\title{
Study on the Characteristics and Cultivating Path of the Industry-University Symbiotic Networks: Based on the Small-World Network Model and the Theory of Symbiosis
}

\author{
Zhenquan Cao ${ }^{1}$, Leiyong Zhang ${ }^{1}$, Feng Feng ${ }^{2} \&$ Yuneng $\mathrm{Du}^{1}$ \\ ${ }^{1}$ The School of Public Affairs, University of Science and Technology of China, China \\ ${ }^{2}$ School of Management, University of Science and Technology of China, China \\ Correspondence: Yuneng Du, The School of Public Affairs, University of Science and Technology of China, \\ China. E-mail: duyuneng@gmail.com
}

\author{
Received: October 11, 2012 Accepted: October 20, 2012 Online Published: December 31, 2012 \\ doi:10.5539/ass.v9n1p15 \\ URL: http://dx.doi.org/10.5539/ass.v9n1p15
}

\begin{abstract}
This Paper proposed the concept of industry-university symbiotic networks based on the analysis of the network and symbiotic characteristic of the cooperation of industry, universities and research institutions. The information, material and energy exchange rate, the degree of concentration and the generation and distribution mechanism of the symbiotic energy of the industry-university symbiotic networks were analyzed. We pointed out that increasing the scale of the network, optimizing the symbiotic interface, necessarily breaking and re-bonding the links and promoting the distribution of the symbiotic energy were the most fundamental means of enhancing the ability of industry-university symbiotic networks. At last this paper drew a path map of cultivating the industry-university symbiotic networks.
\end{abstract}

Keywords: industry-university symbiotic networks, small world network, symbiosis, characteristics, path of cultivating

\section{Introduction}

Driven by the interests, universities and enterprises will use their own resources to collaborate complementary economic and social activities. The cooperation between universities and enterprises can speed up technological progress and improve the efficiency of scientific achievements, and play an important role in the national economic and social development. How to speed up the cooperation between universities and enterprises, enhance the level of cooperation has become an important issue.

With the deepening of the cooperative relationship between universities and enterprises, more and more universities and enterprises are involved in, the level of cooperation are increasing, and the cooperation appeared like a network. On the other hand, the cooperation between them, in essence, is also the pursuit of a symbiotic relationship (Sheng, 2007). Many researchers study on the cooperation networks of the universities and the enterprises, for examples, Feng et al(2008)used small world network model to analyze the structural characteristics of the cooperative innovation networks and put forward targeted cultivate mechanism; $\operatorname{Li}(2010)$ studyed the structure and cultivate mechanism of the cooperation networks of the universities and the enterprises via scale-free network model; Han(2006) studied characteristics and governance mechanisms of regional research cooperation innovation networks via complex scientific theories; Jiang et al(2007)used modern field theory to analysis the characteristics of the network of technology innovation. Those scholars mainly research cooperation network characteristics from a network perspective, but they did not involve the formation mechanism of the cooperative relationship between the nodes. Si et al(2009)points out that the cooperation relationships of the universities and the enterprises are symbiosis relationships, and the theory of symbiosis is a appropriate perspective of studying on the cooperation of enterprises and universities. It provides a whole new way of thinking for us to study the I-U (Short for Industry-University) symbiotic network.

The author illustrated the formation and cultivated the mechanism of the I-U symbiotic networks via the Small World Network Model and the Theory of Symbiosis. This paper claimed that grasping the network characteristic of the cooperation of universities and enterprises can take full advantage of multiplier effect of excellent network and enhance the level of cooperation. The symbiotic theory provides a new perspective for the optimization of 
cooperation of universities and enterprises with the value of the methodology.

\section{Industry-University Symbiotic Networks (IUSN)}

The Symbiotic networks first appeared in the fields of the biology,and was refer to the network forms by biological populations which live together according to a certain pattern of interdependence and interaction. They have the characteristics of the co-existence and co-evolution. With the deepening of cross-disciplinary research, the symbiotic network theory was gradually expanded to the fields of economics and sociology. A typical application of symbiotic network theory in the economics is the proposal of eco-industrial park,the enterprises in the park transfer material and energy or exchange knowledge and resources via the network, form a symbiotic relationship of long-term cooperation (Mirata, and Emtairah, 2005).

The author introduced the symbiotic theory into the research of the networks of universities and enterprises and proposed the concept of industry-university symbiotic networks (IUSN). Through referencing the study of Zhu et al (2003), and involving the symbiotic theory into this research, the author define the industry-university symbiotic networks as "System or joint institutions with networks conformation which form by the symbiotic activities of researching, developing or industrialization of universities and enterprises via different symbiotic models on the symbiotic interface they form in certain symbiotic environment". The IUSN have the characteristic of small world network and node-symbiosis simultaneously.

The concept of social network was proposed in 1950s. A lot of statistical data show that the social network has the features of complex network. According to the information transfer characteristics of the society, Mitchell(1969)define the social network as a certain group of connections of a certain person, and he claimed that these connections can be regard as a whole in interpretation of personal social behavior in the network. Watts and Strogatz(1998)proposed the small world network model based on the research of the characteristics of human social networks and complex networks. Information transfer process of actors in the networks is also a kind of social behavior, and the networks have the characteristics of small-world. Transfer of information, such as technology or knowledge, exists in cooperation between universities and enterprises, so the network of universities and enterprises also have characteristics of small-world. On the other hand, two types of symbiotic nodes, which refer to the universities and enterprises, exist in the industry-university symbiotic networks. Two types of nodes show quality parameters compatible, exchange material and energy via fund and technology. They form stable connection and symbiotic relationship of mutually beneficial coexistence, division of work and complementary advantages (Kumar, 1994). With the exacerbation of the I-U cooperation, it must develop towards direction of the symbiotic network: a characteristics of small-world and at the same symbiosis exists in nodes of network (Kleinberg, 2004).

In I-U Symbiotic Networks, the symbiotic unit includes enterprises, universities which may be directly involved in the symbiotic activities. Government policy environment, economic environment, information environment, space environment and social norms environment constituted the symbiotic industry-university symbiotic network. Symbiotic units in the networks carry out symbiotic activities on the tangible or intangible symbiotic interface. Based on the definition above, we can draw a conceptual model of industry-university symbiotic networks as show in figure 1.

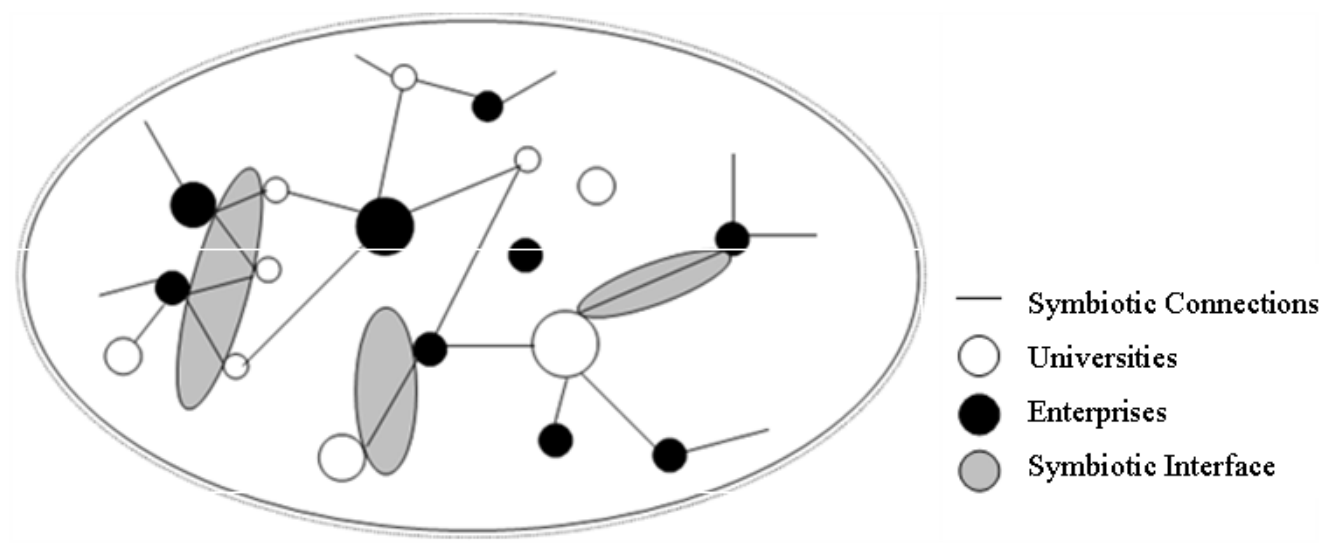

Figure 1. Conceptual model of industry-university symbiotic network

In figure 1, the black perfect circles represent enterprise symbiotic unit and white perfect circles represent universities. The diameters of perfect circles represent the strength of symbiosis unit. The gray ellipses represent 
intermediaries or other media symbiotic interface. The largest ellipse represents the symbiotic environment of the symbiotic network. Solid lines connecting lines represent symbiotic relationships. One symbiotic unit may take part in the symbiotic relationship, or in may do not participate in any symbiotic activities. Symbiotic units can do participate in symbiotic activities on media interface such as technical innovation alliances or intermediaries, and they can also do it on a no-media interface (directly connected in figure .1) such as technology port or technology standard. One symbiotic unit may have symbiotic relationship with one or more other symbiotic units. The symbiotic relationships include parasitic, partial commensalism, mutualism and integration symbiotic.

\section{Analysis of Characteristics of Industry-University Symbiotic Networks}

\subsection{Overview of the Small-World Network Model}

The small-world phenomenon can be traced back in the 1960s, when social psychologist Mitchell(1969) Studied on shortest path of social network in the USA. Based on the model of social network, Watts(1998)proposed the small-world network model in his theoretical research of regular networks and random network. This kind of network changes from regular network to random network by adjusting a variable. The construction algorithm of small-world is: first begin with a ring network with $\mathrm{N}$ nodes, then each node send out $K$ connections to its adjacent nodes, and $N>>K>>\ln (N)>>1$. Each connection has a probability of $p$ that changes its purpose connection and reconnects its side, and no duplicated connections will appear. The network will form $p N K / 2$ long connection to joint a node to another further one. Change the value of $\mathrm{p}$, the network will be changed from a regular network $(p=0)$ to a random network $(p=1)$ (Feng et al, 2006). Watts think that many networks in practice is neither completely regular nor completely random, so the appearance of small-world network has important practical significance.

The theory of small-world network was constantly improve and had been used to solve large kinds of real problems. Currently, as a new scientist results of complexity science and complex networks, the small-world network model is widely used in areas such as Information Network Control(Albert and Barabasi, 2002), HIV Disease Control(Liljeros et al, 2001), network dynamics of biological macromolecules(Jeong et al, 2001), and has achieved great success. The I-U network show typical feature of small-world, and the 0ptimization of its structure will play a role of multiplication, so we may use the small-world network model to research on the I-U network. In small-world network, Characteristics Path Length (CPL), Clustering Coefficient $(C)$ and Probability (p) of reconnect broken connections can be used to describe the characteristics of network. Characteristics Path Length represent the average shortest distance between two nodes in the network, and it can measure the whole characteristics of the network. Clustering Coefficient can describe the partial features of the network, and measure whether there are relatively stable subnets existing in the network. Probability of reconnect broken connections can measure the probability level that one node change its target connection node and reconnect its original side.

\subsection{Overview of Symbiotic Theory}

The conception of "symbiosis" was first proposed by German biologist Anion De Bary in 1879, and referred to the co-existence, co-evolution relationship that formed by two or more living creatures according to some certain mode for the need to survive. At past, the symbiosis theory was usually used to explain biological relationship of co-existence and co-evolution in biosphere. In recent years, the theory of symbiosis is gradually expanding to various fields such as sociology and economics. One typical application of the theory of symbiosis is the industrial symbiosis park, enterprises in the park take part in the symbiotic activities (Mirata et al, 2005; Zhu et al,2007). Yuan(1998)constructed the analytical framework of the theory of symbiosis in the social science and do research on the small economic via theory of symbiosis. He claimed that, the symbiosis is the relationship that the symbiotic unit forms according to some certain modes in some certain environment. He pointed out the description and basic principles of symbiosis.

Generally, the symbiotic relationship are usually affected by symbiotic interface, symbiotic energy and the qualitative parameters compatibility. Symbiotic interface is the contact ways and mechanisms of symbiotic units, or the media, passage and carrier of the transference of material, information and energy. It is the basis of formation and development of symbiotic relationships and it will affect the relationships directly. Symbiotic energy is the new energy that the symbiotic activities produce. Generation and distribution of the symbiotic energy will affect the stability of symbiotic relationships. Qualitative parameters compatibility can be used to measure the potential tightness of the cooperation of the symbiotic units. Generally, Symbiotic units with higher qualitative parameters compatibility will form stable and close symbiotic relationships more easily, and they will produce bigger symbiotic energy. 


\subsection{Characteristics Analysis of Industry-University Symbiotic Networks}

The most important characteristics of I-U symbiotic network are the network characteristics and the symbiotic characteristics. The symbiosis is the basis of the connections in the network and the network is the form of Multi-node symbiosis.

\subsubsection{Exchange Frequency of Substance, Information and Energy}

In the I-U symbiotic networks, the nodes are the enterprises and the universities, and we call them symbiotic unit node. Substance, information and energy (what can be funds, technology, talented persons and equipments) are exchanged between the enterprise symbiotic unit node and the university symbiotic unit node. We may assume that connected graph of the Industry-University Symbiotic Network is G. Then according to the small-world network model, the Characteristics Path Length CPL of G can be measured by

$$
\mathrm{CPL}(\mathrm{G})=\frac{1}{\mathrm{~N}} \frac{1}{\mathrm{~N}-1} \sum_{i \neq j \in G} d_{i j}
$$

$N$ is the number of symbiotic unit nodes in the I-U symbiotic network and $d_{i j}$ means the shortest path length of node $i$ and node $j$. The path length can be the geographical distance or the social distance such as the cooperation relationship, closeness degree between nodes. The exchange frequency of substance, information and energy is inversely proportional to the shortest distance between nodes (Deng et al, 2005) and is proportional to the qualitative parameters compatibility. Qualitative parameters compatibility can measure the degree of potential symbiotic closeness of two nodes, that is extent to which the symbiosis between the two symbiotic units. Qualitative parameters compatibility has a close relationship with the utilization degree of transferred technology. If the technology can be used perfectly the qualitative parameters compatibility is close to 1 . If the technology can't be used at all, then the qualitative parameters compatibility is close to 0 . We assume that the exchange frequency of the substance, information and the energy is $\varepsilon_{\mathrm{ij}}$, qualitative parameters compatibility is $z_{i}$, then we have

$$
\varepsilon_{i j}=k z_{i j} / d_{i j}
$$

$\mathrm{K}$ is a constant. Then we have that the characteristics path length is

$$
\mathrm{CPL}(\mathrm{G})=\frac{1}{\mathrm{~N}} \frac{1}{\mathrm{~N}-1} \sum_{i \neq j \in G}\left(k z_{i j} / \varepsilon_{i j}\right)
$$

We can know from the equation (2) and equation (3) that the exchange or communication frequency of the network is approximately inversely proportional to the CPL of the IUSN. So if we lower the CPL of the network, the frequency of exchange or communication will increase. We can also know from the equation that increase the number of nodes $\mathrm{N}$ or the qualitative parameters compatibility $\mathrm{Z}$ can lower the CPL. The addition of nodes can increase the possibility of potential shorter Shortest Path, and then reduce the CPL. Increase of the qualitative parameters compatibility can lower the degree of difficulty and increase the level of activity, and then promote the upgrading of the frequency of exchange or communication. There are two ways to increase the qualitative parameters compatibility: the first way is to improve symbiotic interface such as the technical interface, and the second one is to make full use of the characteristics of disconnected and reconnect of the small-world model. Promote the connection of nodes with higher qualitative parameters compatibility and avoid or broke the connection of nodes with lower qualitative parameters compatibility.

\subsubsection{Analysis of Clustering Coefficient}

With the scale developing of the IUS (Industry-University Symbiotic), the stability becomes more and more important. The optimization of clustering environment plays an important role in the area of technology transfer and the S.T. Cooperation. In the small-world network model, the Clustering Coefficient can be used to measure the average agglomeration degree of IUSN. Assume the real connections in the network graph $G$ is $e$ and the most probably connections is $E$. Then for any node i in the network, its partial clustering coefficient can be described as

$$
C\left(G_{i}\right)=\frac{e}{E}=\frac{e}{\lambda_{i}\left(\lambda_{i}-1\right) / 2}
$$

Gi represent the sub-graph of node $i, \lambda_{\mathrm{i}}$ means the number of nodes that have connections with node $i$. Then the Clustering Coefficient of IUSN is the average value of the Clustering Coefficient of all nodes, as: 


$$
C(G)=\frac{1}{N} \sum_{i \in G} C\left(G_{i}\right)
$$

According to the equation (4) and equation (5), we know the Clustering Coefficient of IUSN is:

$$
C(G)=\frac{1}{N} \sum_{i \in G} \frac{e}{\lambda_{i}\left(\lambda_{i}-1\right) / 2}
$$

Different nodes has different Clustering Coefficient, their places in the network are different. Node with high Clustering Coefficient has a central position in the network and it has Strong radiation capacity, control and the ability of intermediaries to other nodes. When the connections of each node $\lambda_{i}$ increase, the clustering coefficient of this node and IUS will increase synchronously. So the disconnected and reconnect of nodes is important way of increasing the clustering coefficient, and then optimize the function of IUSN.

\subsubsection{The Production and Distribution of Symbiotic Energy}

The production of symbiotic energy is the essential reason of symbiosis. Symbiotic energy can be the increase of profit in enterprises or the increase of scale of symbiotic units. The symbiotic activities can produce additional energy, so the enterprises and universities would like to take part in proactively. The production and distribution of symbiotic energy will affect the stability of the IUSN. Assume that the symbiotic interface characteristics coefficient is $\lambda, \delta_{\mathrm{ij}}$ is the symbiotic degrees of two nodes, then the full nodes symbiotic degree is

$$
\delta_{\mathrm{G}}=\frac{1}{\lambda} \sum_{i}^{N} \delta_{i j}
$$

And the symbiotic energy of the IUSN is

$$
E=f\left(\delta_{G} / \lambda\right)
$$

So, for node $\mathrm{m}$ and node $n$, the energy they obtain are Em and En, $k$ is symbiotic energy distribution coefficient, $K$ is the stable symbiotic energy distribution coefficient, then

$$
k=E_{m} / E_{n}, k=(1+\alpha) K
$$

$\alpha$ means the deviation degree of the real situation from the ideal state. Optimizing the symbiotic interface and increasing the symbiotic degree can increase the symbiotic energy of the whole network. Symbiotic degree is affected by the qualitative parameters compatibility, clustering coefficient and the characteristics of the symbiotic interfaces. $k$ will affect the openness and stability of the network, the more the $k$ is close from $K$, the more stable the network will be.

\subsubsection{Cultivation Path of IUSN}

The author finds that the most important cultivation ways of IUSN are optimizing the network structure and consolidate the symbiotic relationship. We can design the path graph of how to cultivate IUSN.

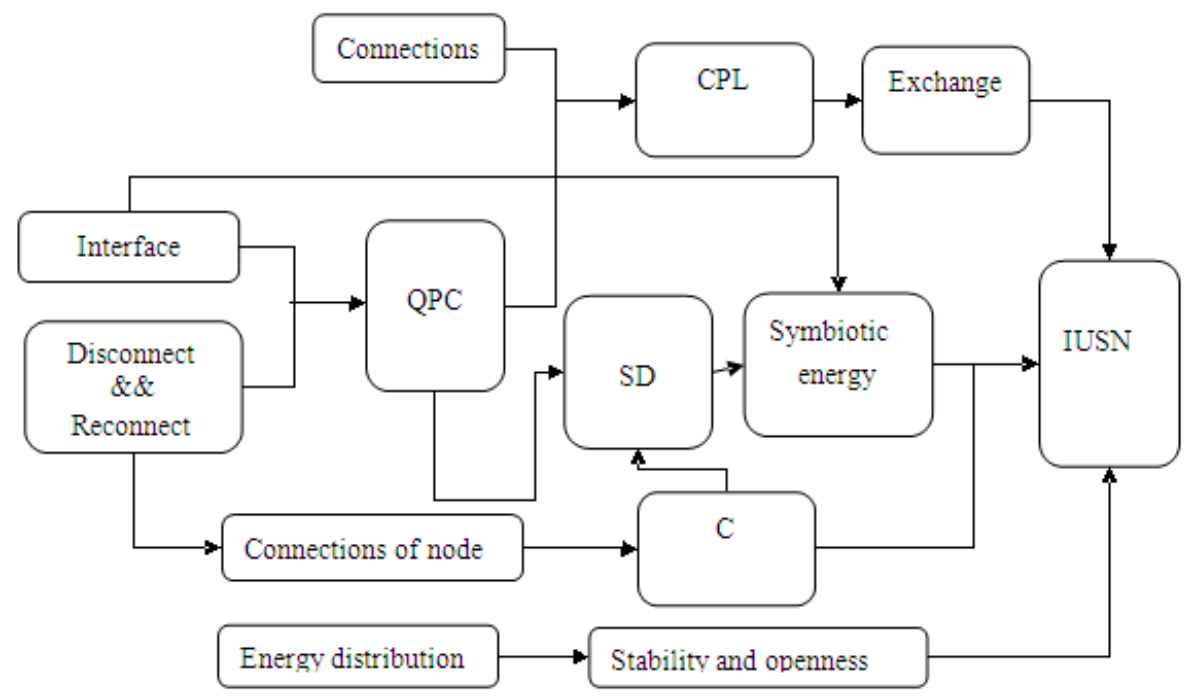

Figure 2. Path graph of how to cultivate IUSN

In figure 2, QPC means qualitative parameters compatibility and SD means symbiotic degree. To increase the function of the IUSN, we can increase the communication of the nodes, the symbiotic energy, the Clustering 
Coefficient or the stability and openness of the IUSN. Upgrade the qualitative parameters compatibility and the number of notes can lower the CPL, and then increase the communication frequency. Optimizing the symbiotic interface and necessary disconnection or reconnection can improve the qualitative parameters compatibility. In addition, the improvement of the qualitative parameters compatibility will increase the symbiotic degree and then plus the symbiotic energy. Disconnection and reconnection can improve the connections of each node, then clustering coefficient will rise. The rise of clustering coefficient will affect the symbiotic degree. Optimize the symbiotic energy distribution can improve the stability and openness of the IUSN. Improve the scale, optimize the symbiotic interface, necessary disconnect and reconnect of the connections and ameliorate the distribution of symbiotic energy are the most directly ways of Cultivating the IUSN.

According to the analysis before, the author put forward some policy suggestions of cultivating the IUSN :(a)Improve the openness of the IUSN, encourage more universities and enterprises taking part in the IUS via positive incentives;(b)Optimize the symbiotic interface. One hand, the governments should increase the construction of the intermediaries and technology transfer center, promote the distribution of the resources, and on the other hand the enterprises and universities should make the technical standards and interfaces more standardized, and then the technology of the universities can be fully used by the enterprises.(c)Necessary disconnection and reconnection. In practice, the nodes in the network must continue correct poor cooperation correction to the high-quality cooperation, and improve the performance of the whole network.(d)Improve the energy allocation. The governments should establish and improve the guide policy on distribution of benefits and guide reasonable distribution of the fruits of innovation and cooperation.

\section{Conclusion}

The cooperation of Industry-University shows characteristics of small-world and symbiosis. The optimization of the IUSN can promote technological innovation and then speed the economic development. This paper put forward the concept of Industry-University Symbiotic Network based on the small-world network model and the symbiotic theory. The author points out the cultivate path of the IUSN. Small-world network can grasp macroscopic characteristics of IUSN (Peng et al, 2011) and the symbiotic theory can microscopic mechanism of cooperation. The theory of IUSN provides a new thinking of study on the modern technology alliance or innovation system, show strong theoretical significance and practical value.

\section{References}

Albert, R., \& Barabasi, A. L. (2002). Statistical mechanics of complex networks. Reviews of Modern Physics, 74, 47-97. http://dx.doi.org/10.1103/RevModPhys.74.47

Deng, D., Li, N., \& Tian, H. (2005). Analysis on NPD Team Communication Network Based on Small-world Network. $R \& D$ Management, 4, 83-86.

Feng, F., \& Wang, L. (2008). Analysis on How to Cultivate Collaborative Innovation Network of Industry-University-Research Institution-Based on Small-world Network Model. China Soft Science, 11, $82-86$.

Feng, F., Zhang, R., \& Yan. W. (2006). Analysis on Interfirm Innovation Network Based on Small-world Network Model. Science of Science and Management of S. \& T., 9, 87-91.

Han, Z. (2006). The Study of Regional Innovation Networks in the view of Science of Complexity. Economic Problems, 9, 11-13.

Jeong et al. (2001). The Large-Scale Organization of Metabolic Networks. Nature, 6804, 651-654.

Jiang, J., Dang, X., \& Liu, L. (2007). Research on the Characters of Knowledge Measurement of Techno-innovation Network Based on Modern Field Theory. China Soft Science, 3, 22-29.

Kleinberg J. (2004). The small-world phenomenon and decentralized search. SIAM News, 37, 1-2.

Kumar, N. (1994). Industry-university symbiosis. International Journal of Engineering Education, 10, 127-132.

Li, X. (2010). Based on the scale-free network perspective research cooperation network structure, functions, and Cultivation Mechanism. University of Science and Technology of China Press: Hefei.

Liljeros et al. (2001). The Web of human Sexual Contacts. Nature, 6840, 907-908. http://dx.doi.org/10.1038/35082140

Mirata, M., \& Emtairah, T. (2005). Industrial symbiosis networks and the contribution to environmental innovation: The case of the Landskrona industrial symbiosis programme. Journal of Cleaner Production, 13, 993-1002. http://dx.doi.org/10.1016/j.jclepro.2004.12.010 
Mitchell, J. C. (1969). Social networks in urban situations. Man-chester: Manchester University Press.

Peng, W., \& Fu, Z. (2011). Review and Prospect of Alliance Portfolios based on Social Network Perspective. Forum on Science and Technology in China, 8, 93-98.

Sheng, Y. (2007). The emergence and development of the technology alliance - a symbiosis of Hermeneutics framework. Forum on Science and Technology in China, 1, 90-93.

Si, S., Cao, Z., \& Feng, F. (2009). Research on the Cooperation Mechanism of Institutions and Enterprises-A Symbiotic Theory and Analytical Framework. Science of Science and Management of S. \& T., 6, 15-19.

Watts, D. J., \& Strogatz, S. H. (1998). Collective dynamics of small-world networks. Nature, 393, 440-442. http://dx.doi.org/10.1038/30918

Yuan, C. (1998). Symbiosis Theory - On small economies. Peking:Economic Science Press.

Zhu, G., \& Peng, Y. (2003). The Research on Organization Mode and Operation Mechanism of Innovation Network in Cooperation of Industry University and Research Institute. Soft Science, 4, 49-52.

Zhu, Y., Qi, Z., \& Fang. L. (2007). Review of Research on industrial symbiosis theory. Industrial Technology \& Economy, 12, 91-94. 